\title{
O ensino da medicina do trabalho nos cursos de graduação médica no Estado do Paraná
}

\author{
The teaching of occupational medicine in undergraduate \\ medical courses in the state of Paraná, Brazil
}

\author{
Guilherme Augusto Murta1, Márcio José de Almeida²
}

\begin{abstract}
RESUMO | Contexto: O conteúdo de Medicina do Trabalho é importante na educação médica, independente da especialidade a ser escolhida, devido à relevância do tema. Do ponto de vista de organizações internacionais, da Legislação Brasileira, do Sistema Único de Saúde e do Conselho Federal de Medicina, a Medicina do Trabalho é entendida como conteúdo esperado para a prática profissional dos médicos. Objetivo: Analisar como está sendo o ensino de temas relacionados à Medicina do Trabalho nos cursos de graduação médica no Estado do Paraná. Métodos: Estudo exploratório com abordagem predominantemente quantitativa. O projeto foi aprovado pelo Comitê de Ética da Faculdades Pequeno Príncipe. Um questionário padrão, validado por especialistas, foi elaborado e dirigido para oito instituições de ensino superior (IES) a fim de pesquisar aspectos referentes ao ensino durante a graduação médica. Resultados: Todos os destinatários responderam ao questionário. A utilização de metodologias ativas de ensino foi verificada em $75 \%$ das escolas médicas. Em apenas metade das IES a disciplina de Medicina do Trabalho estava disponível. Houve identificação de reduzida carga horária (teórica e prática) e poucos temas de saúde ocupacional eram abordados. Paralelamente, o número de periódicos sobre o assunto deixa muito a desejar. Conclusão: As evidências apontam que a Medicina do Trabalho é ensinada de forma insuficiente nos cursos de graduação médica no Estado do Paraná. Seria desejável que pesquisas análogas fossem elaboradas incluindo IES de todo o território brasileiro para, então, possibilitar conclusão representativa nacional.
\end{abstract}

Palavras-chave I ensino; medicina do trabalho; educação superior.

ABSTRACT | Context: The Occupational Medicine content is important in medical education owing to the relevance of the subject, regardless of the chosen specialty. From the perspective of many international organizations, Brazilian legislation, Unified Health System, and Federal Council of Medicine, the Occupational Medicine is a content that is expected for the physicians' professional practice. Objective: To analyze how Occupational Medicine contents are being taught in undergraduate medical programs in the state of Paraná, Brazil. Methods: This is an exploratory study, which mainly applied a quantitative approach. The project was approved by the Ethics Committee of the Faculdades Pequeno Príncipe. A standard questionnaire was developed and validated by experts. This questionnaire was directed to eight higher education institutions (HEI) to investigate aspects of teaching in their undergraduate medical programs. Results: All recipients responded to the questionnaire. Active teaching methods were used in $75 \%$ of medical schools. Only 50\% of HEI had the discipline of Occupational Medicine available. Teaching hours were reduced (theoretical and practical) and few occupational health issues were addressed. Concomitantly, the number of journals on the subject is inadequate. Conclusion: The evidence suggests that Occupational Health is taught inadequately in undergraduate medical programs in the state of Paraná. It would be important to develop similar studies including HEI throughout Brazil to enable nationally representative conclusions. Keywords I teaching; occupational medicine; education, higher. 


\section{INTRODUÇÃO}

As doenças e os acidentes de trabalho constituem graves realidades que chamam a atenção da sociedade pelos prejuízos que causam à saúde da população. Segundo a Organização Internacional do Trabalho $(\mathrm{OIT})^{1}$, estimativas apontam para um total de 2,35 milhões de acidentes de trabalho mortais a cada ano. Isso pode ser traduzido para aproximadamente 1 morte e 115 acidentes a cada 15 segundos. Dessas mortes, $14,9 \%$ decorreram de acidentes e $85,1 \%$ foram causadas por enfermidades variadas relacionadas ao trabalho. Assim, apesar dos acidentes despertarem maior atenção imediata, as doenças do trabalho resultaram nas patologias ocupacionais mais prevalentes. Estas necessitam de um olhar particular, justamente por serem de difícil identificação, situação em que o médico, com conhecimento adequado sobre Medicina do Trabalho, ocupa posição fundamental quanto ao diagnóstico e ao tratamento.

Muitas referências destacam a relevância do processo saúde-doença ocupacional. A OIT aborda o assunto nas Convenções 155 e $161^{2}$. Também a Organização Mundial da Saúde $(\mathrm{OMS})^{3}$ recomenda a implementação das boas práticas visando à proteção dos trabalhadores, à necessidade de normatização e ao estímulo de uma política nacional de saúde e segurança no trabalho.

A legislação brasileira prevê diversos requisitos referentes à saúde e à segurança no trabalho. A Norma Regulamentadora $\mathrm{n}^{\circ} 7^{4}$ tem ações voltadas especificamente para a saúde ocupacional e aponta a necessidade de implantação do Programa de Controle Médico de Saúde Ocupacional (PCMSO). Com essa regulamentação todos os trabalhadores com vinculação regida pela Consolidação das Leis do Trabalho (CLT) têm, de forma compulsória, de ser atendidos em saúde ocupacional de forma preventiva com um programa estruturado a ser desenvolvido por médico do trabalho. Entretanto, esse profissional pode delegar a outro médico, mesmo que não seja especialista, a elaboração de exames (item 7.3.2 da NR-7). Isso aponta para a possibilidade de médico generalista estar diretamente inserido no PCMSO e, para tanto, necessita estar provido com o devido conhecimento desde o momento da graduação.

No âmbito do Sistema Único de Saúde (SUS), a Lei no 8.080, de 1990, Lei Orgânica da Saúde ${ }^{5}$, indica que o cuidado com os trabalhadores deve estar incluído na política de Estado com ação do SUS. No artigo 13 isso fica claro: "A articulação das políticas e programas, a cargo das comissões intersetoriais, abrangerá, em especial, as seguintes atividades: (...) VI - saúde do trabalhador”.

No âmbito do Conselho Federal de Medicina (CFM), há destaque para assistência médica ao trabalhador. O Código de Ética Médica ${ }^{6}$ prevê situações com relação à saúde dos trabalhadores, nos princípios fundamentais (item XII): “O médico empenhar-se-á pela melhor adequação do trabalho ao ser humano, pela eliminação e controle dos riscos à saúde inerentes às atividades laborais". Além disso, veda ao médico "deixar de esclarecer o trabalhador sobre as condições de trabalho que ponham em risco sua saúde, devendo comunicar o fato aos empregadores responsáveis" (artigo 12) e "deixar de esclarecer ao paciente sobre as determinantes sociais, ambientais ou profissionais de sua doença” (artigo 13).

A Resolução do CFM no 1.488 , de $1998^{7}$, reforça o conceito e indica que entre as atribuições esperadas do médico, independente da especialidade, está a de zelar pela promoção, pela prevenção e pela recuperação das saúdes coletiva e individual dos trabalhadores. Assim, do ponto de vista ético, o médico também responde pelos conceitos da Medicina do Trabalho entre suas premissas de atuação, sendo, portanto, imprescindível que o tema seja previsto e ensinado durante a graduação médica e, por isso, contido na programação curricular.

Entretanto, nas Diretrizes Curriculares Nacionais do Curso de Graduação em Medicina ${ }^{8}$, surpreendentemente, não há referência direta ao ensino da Medicina do Trabalho, abordando-a apenas de forma tangencial, como na seção "Formulação de Hipóteses e Priorização de Problemas": “(...) prognóstico dos problemas do paciente, considerando os contextos pessoal, familiar, do trabalho, epidemiológico, ambiental e outros pertinentes". Sendo assim, a dimensão trabalho é indicada, nesse trecho, como fator relevante diante da conduta do médico quando se trata do prognóstico do paciente.

Em relação a competências médicas, no que se refere à prática médica, as Diretrizes Curriculares Nacionais do Curso de Graduação em Medicina ${ }^{1}$ definem ${ }^{8}$ :

(... e é compreendida como a capacidade de mobilizar conhecimentos, habilidades e atitudes (...) que se apresentam à prática profissional, em diferentes contextos do trabalho em saúde, traduzindo a excelência da prática médica, prioritariamente nos cenários do SUS. 
No contexto da Medicina do Trabalho, para Dias et al. ${ }^{9}$, o profissional médico competente deve estar atualizado, saber organizar criticamente informações e dominar habilidades para buscar a "promoção, manutenção, restauração da saúde e de uma melhor qualidade de vida das pessoas sob sua responsabilidade". Indica que o referencial das competências é utilizado não apenas como parâmetro para graduação médica, mas também para orientar processos de formação de especialistas e educação continuada.

O ensino de conteúdo da Medicina do Trabalho mostra-se importante na prática profissional, sendo utilizado para o diagnóstico e consequente adequação na terapêutica de patologias que podem ter correlação com trabalho. A identificação da influência do trabalho na saúde foi evidenciada por Bernardino Ramazzini ${ }^{10}$ há mais de 300 anos: "Um médico que atende um doente deve informar-se de muita coisa (...). A estas interrogações devia acrescentar-se outra: que arte exerce?”. Assim, fatores relacionados ao trabalho devem ser considerados parte integrante da anamnese médica e ensinados na graduação.

Nesse sentido, de acordo com De Lucca e Campos ${ }^{11}$, a inclusão de fatores laborais devem ser aprofundados na anamnese:

(...) o grande desafio consiste na correta associação da doença com o trabalho que raramente se decorrerá da utilização exclusiva de determinado teste ou procedimento laboratorial, exigindo a busca de outras fontes de informação e, fundamentalmente de uma abrangente e apropriada coleta da história do paciente.

Apesar de essas referências indicarem a importância do assunto, na literatura pesquisada encontram-se muitos indícios de que a deficiência no ensino da Medicina do Trabalho parece ser uma falha pedagógica nas Instituições de Ensino Superior (IES) das diversas nações do globo.

No Reino Unido, Williams, Wynn e Whitaker ${ }^{12}$ referendam que os médicos em formação deveriam obrigatoriamente adquirir conhecimentos de saúde ocupacional. Paradoxalmente, em pesquisa ${ }^{12}$ respondida por $66 \%$ das escolas de Medicina do Reino Unido, menos da metade dessas (48\%) tinham a Medicina do Trabalho como disciplina formal.

$\mathrm{Na}$ Espanha ${ }^{13,14}$ e no México ${ }^{15}$, estudos também evidenciam uma carência na formação de Medicina do Trabalho durante a graduação. Em pesquisa respondida por 135 universidades da Europa ${ }^{16}$, houve um levantamento que obteve como média de horas dedicadas formalmente à Medicina do Trabalho apenas 25,5 horas, sendo que $52 \%$ das IES responderam dedicar tempo igual ou menor a 20 horas.

Também nos cursos de Medicina na Turquia, de acordo com Cimrin, Albayrak e Tabak ${ }^{17}$, não havia, de modo geral, uma educação programada e estruturada durante a graduação médica. Concluiu-se que tanto a carga horária quanto a qualidade do ensino da Medicina do Trabalho eram insuficientes.

Em 2015, foi publicado um estudo feito com abrangência global em 21 universidades e 1.985 estudantes ${ }^{18}$, o qual evidenciava a informação de que os alunos de países subdesenvolvidos tinham um menor interesse quanto à saúde e segurança no trabalho do que os de países desenvolvidos. Apesar disso, outra pesquisa com representantes de diversos países ${ }^{19}$ indicou que após um treinamento compulsório tanto o interesse quanto o conhecimento sobre Medicina do Trabalho foram aprimorados em alunos de graduação em Medicina.

No Brasil, segundo Kawakami et al. ${ }^{20}$, existiam indícios de que o ensino de Medicina do Trabalho nas escolas médicas também era incipiente, demandando maior atenção. Conforme indicação de De Lucca e Kitamura ${ }^{21}$, o aprimoramento do ensino para formar médicos que atendam às necessidades da sociedade seria um desafio permanente para as escolas de medicina. Como já dito, a Medicina do Trabalho estaria inserida nesse contexto, sendo, então, importante fazer parte do escopo curricular.

Ao levar em consideração esses diversos argumentos quanto à indubitabilidade da importância de assuntos de Medicina do Trabalho e o paradoxal indício da lacuna pedagógica desse tema durante a formação médica, conforme consta em referências prévias, este estudo tem o objetivo de analisar como ocorre o ensino da Medicina do Trabalho nos cursos de graduação em Medicina no Estado do Paraná.

\section{MÉTODOS}

Este artigo tomou por base uma pesquisa exploratória com método de estudo descritivo. A abordagem utilizada para a pesquisa teve predominância quantitativa, mas foi utilizada também a avaliação qualitativa.

O projeto da pesquisa foi registrado na Plataforma Brasil e submetido à avaliação do Comitê de Ética da Faculdades Pequeno Príncipe. A aprovação do comitê de ética ocorreu em 14 de setembro de 2015. 
Como ferramenta para coleta de dados foi desenvolvido um questionário, que foi apresentado à apreciação de três especialistas para os apontamentos pertinentes quanto à validação de conteúdo. Após esse retorno, a versão final do questionário padrão foi elaborada e dividida em três dimensões: dados sobre a instituição e sobre o destinatário do questionário; informações sobre o ensino da Medicina do Trabalho; e particularidades referentes aos alunos sob o ponto de vista docente.

Os questionários foram enviados aos coordenadores da disciplina de Medicina do Trabalho do Estado do Paraná. Caso não houvesse disciplina de Medicina do Trabalho na IES, então o coordenador do curso de Medicina teria a atribuição de responder ao questionário.

Nos critérios de exclusão estavam os cursos de Medicina que não tinham turmas formadas. Esse pré-requisito foi baseado no fato de que muitos aspectos poderiam ser adequadamente avaliados apenas quando o tema já tivesse sido abordado e isso poderia ter ocorrido dentro dos seis anos de curso. Assim, das 15 IES do Estado com curso de medicina, oito foram selecionadas.

O questionário padrão foi direcionado ao responsável pelas respostas, em conjunto com o Termo de Consentimento Livre Esclarecido e a autorização do departamento da IES. Após as respostas, os questionários foram tabulados.

\section{RESULTADOS}

Todas as oito IES do Paraná que foram incluídas na pesquisa responderam ao questionário, alcançando assim $100 \%$ de participação.

As instituições com a disciplina formalmente ministrada como Medicina do Trabalho totalizaram 50\%. É importante comentar que nos casos em que as IES não ofertam a Medicina do Trabalho como disciplina obrigatória não existia a possibilidade de o aluno cursá-la de forma facultativa.

A utilização de metodologias ativas de ensino foi apontada como predominante. Estavam presentes em $75 \%$ das IES. Por outro lado, apenas 50\% das IES utilizavam provas práticas em avaliação. Número ainda mais reduzido, de apenas $25 \%$, era o das instituições que ofertavam aulas práticas com acompanhamento de docentes nos ambientes de trabalho.
Foram pesquisados quais assuntos de Medicina do Trabalho eram abordados durante algum momento no curso de medicina nas IES pesquisadas e o resultado está disposto conforme a Tabela 1.Legislação em Saúde do Trabalhador foi conteúdo presente em $75 \%$ das IES. Mais de $50 \%$ das instituições pesquisadas também ministravam os temas de avaliação de riscos ocupacionais, ergonomia e exames médicos ocupacionais. Por outro lado, menos da metade das IES responderam que no currículo havia temas referentes à psicopatologia ocupacional, à toxicologia ocupacional, à dermatologia ocupacional e aos programas de qualidade de vida no trabalho.

Sobre a percepção da relevância da Medicina do Trabalho para a formação profissional, a pesquisa contemplava a pergunta "Assuntos relacionados à Medicina do Trabalho são percebidos como importantes?" (pelos alunos). Apenas uma resposta foi indicada como positiva (12,5\%). Entre as IES que indicavam que a Medicina do Trabalho não era importante aos olhos dos alunos, o questionário solicitava justificativa e apenas três discorreram: "não percebem a importância para o futuro"; "Desconhecimento. Não valorização."; e "não reconhecem na Medicina do Trabalho uma carreira, porém sabemos que o é."

Tabela 1. Assuntos do campo de Medicina do Trabalho conforme quantidade de cursos de medicina estudados. Estado do Paraná, 2016.

\begin{tabular}{lcc} 
Assuntos & $n$ & $\%$ \\
\hline Legislação em saúde do trabalhador & 6 & 75,0 \\
\hline Avaliação de riscos ocupacionais & 5 & 62,5 \\
\hline Ergonomia & 5 & 62,5 \\
\hline Exames médicos ocupacionais & 5 & 62,5 \\
\hline Higiene ocupacional & 4 & 50,0 \\
\hline Pneumopatologia ocupacional & 4 & 50,0 \\
\hline Transtornos músculoesqueléticos & 4 & 50,0 \\
\hline ocupacionais & 3 & 37,5 \\
\hline Psicopatologia ocupacional & 3 & 37,5 \\
\hline Toxicologia ocupacional & 2 & 25,0 \\
\hline Audiologia ocupacional & 2 & 25,0 \\
\hline Dermatologia ocupacional & 2 & 25,0 \\
\hline Programas de qualidade de vida no trabalho & 2 &
\end{tabular}




\section{DISCUSSÃO}

O fato de $100 \%$ das IES do Paraná incluídas na pesquisa terem respondido ao questionário confere aos resultados obtidos uma representatividade com relação aos cursos existentes. Comparativamente, estudos de referência de literatura que buscaram pesquisar o ensino da Medicina do Trabalho tiveram participação menor, como $66^{12}, 44^{16}$ e $30 \%{ }^{20}$.

Apesar do sucesso de participação percentual, as referências literárias comparativas utilizadas foram numericamente superiores, tendo contado com a participação de 21 escolas britânicas $^{12}, 28$ instituições espanholas ${ }^{22}, 48$ instituições brasileiras $^{20}$ e 135 IES europeias ${ }^{16}$.

Apenas 50\% das IES paranaenses ofertavam a disciplina de Medicina do Trabalho durante a graduação médica. Essa proporção mostrou-se inferior a referências de literatura dos estudos europeus $(95 \%)^{16}$ e brasileiros $(94,9 \%)^{20}$, mas curiosamente foi semelhante aos britânicos ${ }^{12}(48 \%)$. $O$ percentual encontrado nas IES paranaenses também foi superior aos 36\% de presença da disciplina de Medicina do Trabalho da pesquisa entre instituições espanholas ${ }^{22}$.
Houve a constatação de que as IES paranaenses que não ofertavam a disciplina de Medicina do Trabalho de forma compulsória tampouco o disponibilizavam de maneira eletiva. Seria esperado, então, que assuntos de tamanha relevância estivessem contidos em outras disciplinas, mesmo que não nominados formalmente como Medicina do Trabalho. Isso não ocorreu, conforme indicam os dados da Tabela 1 .

Causa estranheza que alguns assuntos extremamente relevantes para atuação médica não tenham sido assinalados como conteúdo disponibilizado na graduação, estando presente em apenas 50\% ou menos das IES paranaenses; são eles: audiologia ocupacional, transtornos mentais ocupacionais, toxicologia ocupacional, pneumologia ocupacional, transtornos musculoesqueléticos ocupacionais. $\mathrm{O}$ assunto de reabilitação profissional não foi indicado por nenhuma das IES como tema integrante no currículo de formação médica.

Como forma de possibilitar parâmetro sobre esses achados, o pesquisador realizou comparativos com referências da literatura, conforme disposto na Tabela 2.

Percebe-se que as IES paranaenses geralmente disponibilizam os conteúdos com proporção aquém das referências

Tabela 2. Assuntos da Medicina do Trabalho presentes nos currículos das escolas pesquisadas e em duas referências da literatura. Estado do Paraná, 2016.

\begin{tabular}{|c|c|c|c|}
\hline Assuntos & $\begin{array}{l}\text { Percentual da pre- } \\
\text { sença do conteúdo } \\
\text { nas IES pesquisadas }\end{array}$ & $\begin{array}{l}\text { Percentual de presença do } \\
\text { conteúdo nas IES pesquisa- } \\
\text { das segundo Williams et al. } .^{12}\end{array}$ & $\begin{array}{l}\text { Percentual de presença do } \\
\text { conteúdo nas IES pesquisa- }^{\text {das segundo Gehanno et al. }{ }^{16}}\end{array}$ \\
\hline Legislação em saúde do trabalhador & 75,0 & Não disponível & 85,0 \\
\hline Avaliação de riscos Ocupacionais & 62,5 & 52,0 & 72,0 \\
\hline Ergonomia & 62,5 & Não disponível & 58,0 \\
\hline Exames médicos Ocupacionais & 62,5 & Não disponível & Não disponível \\
\hline Higiene ocupacional & 50,0 & Não disponível & Não disponível \\
\hline Pneumopatologia ocupacional & 50,0 & 81,0 & 89,0 \\
\hline $\begin{array}{l}\text { Transtornos Musculoesqueléticos } \\
\text { Ocupacionais }\end{array}$ & 50,0 & 67,0 & 81,0 \\
\hline Psicopatologia ocupacional & 37,5 & 76,0 & 72,0 \\
\hline Toxicologia ocupacional & 37,5 & 48,0 & 85,0 \\
\hline Audiologia ocupacional & 25,0 & Não disponível & Não disponível \\
\hline Dermatologia ocupacional & 25,0 & 76,0 & 78,0 \\
\hline Programas de qualidade de vida no trabalho & 25,0 & Não disponível & Não disponível \\
\hline Reabilitação profissional & 0,0 & 71,0 & 44,0 \\
\hline
\end{tabular}


comparadas, quando há dado disponível. Não foi incluído na Tabela 2 o estudo de Kawakami et al. ${ }^{20}$, que engloba IES de diversas regiões brasileiras, pois os assuntos não foram abordados nesta publicação.

De acordo com os dados levantados por este estudo, entre as IES que ofertavam a Medicina do Trabalho como disciplina, somente metade avaliava os alunos com provas práticas. Apenas um quarto dos professores acompanhava os alunos em visitas aos ambientes de trabalho, em atividade prática. De acordo com De Lucca e Kitamura ${ }^{21}$, essa prática pedagógica é um diferencial relevante para o aprimoramento da educação em Medicina do Trabalho.

Em relação à carga horária destinada ao assunto de Medicina do Trabalho, obteve-se como resultado, em média, 49,5 horas nas IES paranaenses. Esse número mostrou ser superior àquele contido na informação revelada por Gehanno et al. ${ }^{16}$, o qual indicou a média de apenas 25,5 horas em escolas médicas europeias. Porém o valor foi inferior ao do estudo de Kawakami et al. ${ }^{20}$, que apontou média de 59,5 horas nas IES brasileiras.

Segundo Williams, Wynn e Whitaker ${ }^{12}$, no contexto britânico, as IES estão reduzindo a carga horária destinada ao tema nos últimos anos. Em 2000, apenas 32\% ofertavam mais de 6 horas de aula. O que parecia insuficiente teve achatamento ainda maior: em 2010, 95\% das IES britânicas disponibilizavam menos de 6 horas no currículo para o mesmo tema.

Em relação a aulas práticas, de acordo com Kawakami et al. ${ }^{20}$, a média da carga horária nas IES brasileiras foi apenas de 21,5 horas - mesmo assim bastante superior à média de 12 horas encontrada no presente estudo. Com isso, nota-se que a carga horária, em geral, para assunto de tamanha relevância, é nitidamente baixa tanto no Brasil como nas referências de estudos internacionais.
A falta de importância atribuída a assuntos relacionados à Medicina do Trabalho durante a graduação pode ter reflexos negativos nas pesquisas e publicações científicas sobre o tema. Para verificar essa hipótese e contabilizar o número de periódicos sobre o tema, o pesquisador consultou o portal da Coordenação de Aperfeiçoamento de Pessoal de Nível Superior (CAPES) ${ }^{23}$ na internet, a classificação Qualis-Periódicos, e selecionou a área de avaliação de Saúde Coletiva. A última listagem de classificação disponível é de 2014. Nela estavam contidos 1.177 periódicos nacionais e internacionais. Ao realizar busca pelos títulos, foi identificado que apenas 1,01\% deles ( 12 periódicos) fazia referência à Medicina do Trabalho, o que ratificou a hipótese inicial.

\section{CONCLUSÃO}

De acordo com diversas referências, como OIT, OMS, Legislação Brasileira e CFM, os assuntos relacionados à Medicina do Trabalho apresentam relevância substancial, havendo entendimento da importância para prática profissional dos médicos, independente da especialidade.

Contraditoriamente, os dados encontrados nesta pesquisa e na literatura referenciada mundial apontam que o tema tem sido relegado; isto é, não está adequadamente incluído como objeto de aprendizagem, resultando em uma lacuna pedagógica com sequelas marcantes para a formação médica.

Ao considerar a importância e a abrangência da Medicina do Trabalho, seria desejável que pesquisas análogas fossem elaboradas no futuro incluindo também IES envolvidas na graduação médica de todo território brasileiro para, então, possibilitar uma conclusão representativa nacional.

\section{REFERÊNCIAS}

1. Organização Internacional do Trabalho. Proteção dos trabalhadores num mundo do trabalho em transformação - Debate recorrente sobre o objetivo estratégico da proteção social (proteção dos trabalhadores), Relatório IV. Genebra, 2015.

2. Brasil. Ministério do Trabalho e Emprego. Secretaria de Inspeção do Trabalho. Convenções da OIT. Brasília: MTE, SIT, 2002.

3. Organização Mundial da Saúde. [Internet] Cinco chaves para ambientes de trabalho saudáveis: não há riqueza nos negócios sem a saúde dos trabalhadores [acesso em 2015 mar 12]. Disponível em: <http://www.who.int/occupational_health/5keys_healthy_ workplaces_portuguese.pdf $>$
4. Brasil. Ministério do Trabalho. Secretaria de Segurança e Saúde no Trabalho. [Internet] Portaria no 24, de 29 de dezembro de 1994. Norma Regulamentadora (NR) no 7 [acesso em 2015 maio 25]. Disponível em: <www2.feg.unesp.br/Home/cipa998/normaregulamentadora-7.pdf>

5. Brasil. Presidência da República. Casa Civil. [Internet] Lei n 8.080, de 19 de setembro de 1990. Dispõe sobre as condições para a promoção, proteção e recuperação da saúde, a organização e o funcionamento dos serviços correspondentes e dá outras providências [acesso em 2015 maio 27]. Disponível em: <http:// www.planalto.gov.br/ccivil 03/leis/ I8080.htm> 
6. Conselho Federal de Medicina. [Internet] Código de Ética Médica Resolução CFM no 1931, de 17 de setembro de 2009 (versão de bolso) [acesso em 2015 maio 28]. Brasília: Conselho Federal de Medicina, 2010. Disponível em: <http://www.portalmedico.org.br/ novocodigo/campanha.asp>

7. Conselho Federal de Medicina. [Internet] Resolução nº 1488, de 06 de março de 1998 [acesso em 2015 out 15]. Disponível em: <http:// www.portalmedico.org.br/resolucoes/cfm/1998/1488_1998.htm>

8. Brasil. Ministério da Educação. Conselho Nacional de Educação. Resolução no 3, de 20 de junho de 2014. Institui Diretrizes Curriculares Nacionais do Curso de Graduação em Medicina e dá outras providências.

9. Dias EC, Chiavegatto CV, Faria HP, Rabelo CR. Competências essenciais requeridas para o exercício da Medicina do Trabalho. Curitiba: Associação Nacional de Medicina do Trabalho, 2016.

10. Ramazzini, B. As doenças dos trabalhadores. 2. ed. São Paulo: Fundacentro, 1999.

11. De Lucca SR, Campos CR. A Medicina do Trabalho no mundo contemporâneo: o perfil dos médicos do trabalho, desafios e competências. Rev Bras Med Trab. 2011;9(1):45-7.

12. Williams N, Wynn PA, Whitaker S. Undergraduate occupational medicine tuition in UK schools of medicine. Occup Med. (London). 2011;61(3):152-6.

13. Caballero Zamora MJ, Andani Cervera J, Ligero López E, Ribes Martínez E, Vico Garcerán B, Saus Carreres A. Elárea de conocimiento de Medicina del Trabajo en el grado de Medicina de las Universidades Españolas. Rev Asoc Esp Espec Med Trab. 2013;22(3):135-41.

14. Arturo López A, Ayensa JA, Núñez C, Vicente-Herrero MT. Teaching of occupational medicine in Spanish medical schools. Arch Prev Riesgos Labor. 2007;10(4):188-91.

15. Sánchez-Román FR, Medina-Figueroa AM, Rangel-Zertuche RA, Sánchez-Ramos A. The teaching of occupational medicine in Mexican medical schools. Salud Publica Mex. 2009;51(2):97-103.
16. Gehanno JF, Bulat P, Martinez-Jarreta B, Pauncu EA, Popescu F, Smits $\mathrm{PB}$, et al. Undergraduate teaching of occupational medicine in European schools of medicine. Int Arch Occup Environ Health. 2014;87(4):397-401.

17. Cimrin A, Albayrak S, Tabak L. Position of occupational diseases in medical education in Turkey. Turbek Toraks. 2010;58(2):142-6.

18. Bhardwaj M, Arteta M, Batmunkh T, Briceno Leonardo L, Caraballo Y, Carvalho D, et al. Attitude of medical students towards occupational safety and health: a multi-national study. Int J Occup Environ Med. 2015;6(1):7-19.

19. Russ P, Strümpell S, Carvalho D, Zander S, Smits P, Nowak D, et al. Compulsory teaching of occupational health: impact on attitude of medical students in Brazil and Germany. Int Arch Occup Environ Health. 2012;85(1):81-7.

20. Kawakami EM, Arruda LM, Borguetti FC, Hayashide JM, Albuquerque LC, Queiroz M, Souza FS, Vido RS, Morrone LC. O Ensino de Medicina do Trabalho no Brasil. Rev Bras Med Trab. 2011;9(1):15-25.

21. De Lucca SR, Kitamura S. O ensino da Medicina do Trabalho e a importância das visitas aos locais de trabalho. Rev Bras Med Trab. 2012;10(2):41-8.

22. López AA, Ayensa JA, Núñez C, Vicente-Herrero MT. Asignaturas específicas o relacionadas con la Medicina del Trabajo en las facultades de Medicina españolas. Arch Prev Riesgos Labor. 2007;10(4):188-91.

23. Brasil. Coordenação de Aperfeiçoamento de Pessoal de Nível Superior (CAPES). Plataforma Sucupira. [Internet] Periódico Qualis [acesso em 2016 maio 4]. Disponível em: <https://sucupira.capes. gov.br/sucupira/public/consultas/coleta/veiculoPublicacaoQualis/ listaConsultaGeralPeriodicos.jsf>

Endereço para correspondência: Guilherme Augusto Murta - Rua Ângelo Breseghello, 422, casa 4 - Bairro Alto - CEP: 82840-540 - Curitiba (PR), Brasil. E-mail: guilhermeamurta@hotmail.com 